\title{
Lessons learned from the anesthetic management of patients with narcolepsy
}

\section{Sara Pedrosa1, Pedro Amorim ${ }^{2}$}

${ }^{1}$ Department of Anesthesiology, Centro Hospitalar do Baixo Vouga, Portugal

2Department of Anesthesiology, Intensive Care and Emergency, Centro Hospitalar do Porto, Portugal

\section{Background and Goal of Study}

Narcolepsy is a neurological disorder that affects sleep regulation and is associated with increased risk of perioperative complications. The aim of this study is to systematically review the literature concerning the anesthetic management of patients with narcolepsy.

\section{Materials and Methods}

A search was conducted in the Medline database. We included all studies of patients with narcolepsy submitted to anesthesia. The parameters analyzed were: type of anesthesia, premedication, withdrawal of medication for narcolepsy, use of depth of anesthesia monitors, time for extubation and perioperative complications.

\section{Results and Discussion}

\begin{tabular}{|c|c|c|c|c|c|c|c|c|c|}
\hline Source & $\begin{array}{l}\text { Study } \\
\text { design }\end{array}$ & Anesthesia & Surgical Procedure & Medication for narcolepsy & $\begin{array}{l}\text { Maintainence of } \\
\text { narcolepsy medication } \\
\text { on the day of surgery }\end{array}$ & Premedication & $\begin{array}{l}\text { Depth of anesthesia } \\
\text { monitoring }\end{array}$ & Time for extubation & Complications \\
\hline $\begin{array}{l}\text { Spector et al, } \\
1977(1)\end{array}$ & Case report & General inhalational & Exploratory laparotomy & None & N/A & $\begin{array}{l}\text { Morphine, diazepam, } \\
\text { atropine }\end{array}$ & No & Not mentioned & $\begin{array}{l}2 \text { episodes of sleep paralysis in the } \\
\text { recovery room and } 1 \text { episode of sleep } \\
\text { paralysis } 4 \text { days after surgery }\end{array}$ \\
\hline $\begin{array}{l}\text { Pasteau et al, } \\
1996 \text { (2) }\end{array}$ & Case report & Spinal & $\begin{array}{l}\text { Lumbar herniated disk } \\
\text { surgery }\end{array}$ & Modafinil $300 \mathrm{mg}$ daily & Not mentioned & Diazepam and pethidine & No & N/A & $\begin{array}{l}2 \text { narcolepsy-cateplexy episodes intra- } \\
\text { operatively }\end{array}$ \\
\hline $\begin{array}{l}\text { Mesa et al, } \\
2000 \text { (3) }\end{array}$ & Case report & General intravenous & Excision of a thigh mass & Dextroamphetamine & Yes & No & No & $\begin{array}{l}15 \text { minutes after the end of } \\
\text { surgery }\end{array}$ & None \\
\hline $\begin{array}{l}\text { Fischer et al, } \\
2000(4)\end{array}$ & Case report & General inhalational & Emergency laparoscopy & $\begin{array}{l}\text { Dextroamphetamine } 10 \mathrm{mg} \text {, } \\
\text { amphetamine } 10 \mathrm{mg} \text { daily }\end{array}$ & Yes & Midazolam & No & Not mentioned & None \\
\hline $\begin{array}{l}\text { Dounas et al, } \\
2002 \text { (5) }\end{array}$ & Case report & Epidural analgesia & Labor & $\begin{array}{l}\text { None (stopped modafinil during } \\
\text { pregnancy) }\end{array}$ & N/A & No & No & N/A & None \\
\hline $\begin{array}{l}\text { GGómez- } \\
\text { Garrido et al, } \\
2002 \text { (6) }\end{array}$ & Case report & General inhalational & Aortobifemoral bypass & Methylphenidate and fluoxetine & Yes & No & No & $\begin{array}{l}2 \text { hours after admission to } \\
\text { PACU }\end{array}$ & $\begin{array}{l}\text { Hypnagogic / hypnopompic } \\
\text { hallucinations }\end{array}$ \\
\hline \multirow{2}{*}{$\begin{array}{l}\text { Vieito et al, } \\
2002(7)\end{array}$} & \multirow{2}{*}{$\begin{array}{l}\text { Two case } \\
\text { reports }\end{array}$} & General inhalational & $\begin{array}{l}\text { Reduction of nasal bones } \\
\text { fracture }\end{array}$ & Methylphenidate & Yes & No & No & Not mentioned & No \\
\hline & & $\begin{array}{l}\text { Axillary brachial } \\
\text { plexus block }\end{array}$ & $\begin{array}{l}\text { Osteosynthesis of radius } \\
\text { and ulnar fractures }\end{array}$ & Pemoline & $\begin{array}{l}\text { No, suspended } 7 \text { days } \\
\text { before surgery }\end{array}$ & No & No & N/A & No \\
\hline $\begin{array}{l}\text { Peláez et al, } \\
2004 \text { (8) }\end{array}$ & Case report & General intravenous & $\begin{array}{l}\text { Quadruple coronary artery } \\
\text { bypass grafting }\end{array}$ & $\begin{array}{l}\text { Amphetamine } 10 \mathrm{mg} \text {, modafinil } 300 \mathrm{mg} \text {, } \\
\text { clomipramine } 150 \mathrm{mg} \text {, daily }\end{array}$ & Yes & No & No & $\begin{array}{l}6 \text { hours after end of } \\
\text { surgery in the ICU }\end{array}$ & None \\
\hline $\begin{array}{l}\text { Burrow et al, } \\
2005 \text { (9) }\end{array}$ & $\begin{array}{l}\text { Case series: } \\
10 \text { patients } \\
\text { submitted to } \\
27 \text { surgeries }\end{array}$ & General anesthesia & $\begin{array}{l}10 \text { orthopedic, } 6 \text { ENT, } 5 \\
\text { urologic, } 3 \text { abdominal, } 2 \\
\text { gynecologic, } 1 \text { thoracic }\end{array}$ & $\begin{array}{l}\text { All patients were treated with one or more } \\
\text { of the following: methylphenidate, } \\
\text { methamphetamine, trazodone, doxepin, } \\
\text { amphetamine, pemoline, perphenazine- } \\
\text { amitriptyline, desipramine }\end{array}$ & Yes & Not mentioned & No & $\begin{array}{l}\text { Time from end of surgery } \\
\text { to endotoracheal tube } \\
\text { removal: } 12 \pm 7 \mathrm{~min} \\
\text { (average and SD) }\end{array}$ & $\begin{array}{l}2 \text { cases of hypersomnolence in the } \\
\text { PACU } \\
1 \text { patient complained of excessive } \\
\text { sleepiness in the } 6 \text { months after } \\
\text { general anesthesia }\end{array}$ \\
\hline $\begin{array}{l}\text { Janeiro et al, } \\
2007 \text { (10) }\end{array}$ & Case report & General intravenous & Pyeloplasty & Clomipramine and modafinil & $\begin{array}{l}\text { Continued modafinil, } \\
\text { suspended clomipramine }\end{array}$ & No & Entropy monitor & Extubated in the PACU & None \\
\hline $\begin{array}{l}\text { Pérez et sl, } \\
2007 \text { (11) }\end{array}$ & Case report & General inhalational & Laryngeal microsurgery & Anafranil $25 \mathrm{mg}$ daily & Yes & Diazepam & No & Not mentioned & None \\
\hline $\begin{array}{l}\text { Ozkose et al, } \\
2007 \text { (12) }\end{array}$ & Case report & General intravenous & Bilateral breast reduction & None & N/A & No & BIS ${ }^{\circledast}$ monitor & Not mentioned & None \\
\hline $\begin{array}{l}\text { Staikou et al, } \\
2007 \text { (13) }\end{array}$ & Case report & General inhalational & $\begin{array}{l}\text { Laparoscopic } \\
\text { cholecystectomy }\end{array}$ & None & N/A & No & BIS ${ }^{\circledast}$ monitor & Not mentioned & $\begin{array}{l}\text { Postoperative narcoleptic events } \\
\text { (similar to her usual ones) }\end{array}$ \\
\hline $\begin{array}{l}\text { Doyle et al, } \\
2008 \text { (14) }\end{array}$ & Case report & General inhalational & Hysteroscopy day surgery & Modafinil $300 \mathrm{mg}$ daily & No & No & No & 10 min (laryngeal mask) & None \\
\hline $\begin{array}{l}\text { Dahaba et al, } \\
2009(15)\end{array}$ & Case report & Femoral nerve block & Lower limb surgery & None & N/A & No & $\begin{array}{l}\text { Two BIS monitors for } \\
\text { both hemispheres }\end{array}$ & N/A & $\begin{array}{l}\begin{array}{l}\text { Narcolepsy-cataplexy episode }(2 \mathrm{~h} \\
\text { duration) }\end{array}\end{array}$ \\
\hline $\begin{array}{l}\text { Montcriol et } \\
\text { al, } 2010 \text { (16) }\end{array}$ & Case report & General intravenous & Colonoscopy & $\begin{array}{l}\text { Methylphenidate } 30 \mathrm{mg} \text {, modafinil } 400 \mathrm{mg} \\
\text { daily }\end{array}$ & Yes & No & $\mathrm{BIS}^{\circledast}$ monitor & N/A & None \\
\hline $\begin{array}{l}\text { Morimoto et } \\
\text { al, 2010 (17) }\end{array}$ & Case report & General inhalational & Endoscopic sinus surgery & Modafinil $100 \mathrm{mg}$ daily & Yes & No & $\mathrm{BIS}^{\circledast}$ monitor & 10 minutes & None \\
\hline $\begin{array}{l}\text { Soltanifar et } \\
\text { al, } 2010 \text { (18) }\end{array}$ & Case report & Epidural & Caesarean section & Modafinil $200 \mathrm{mg}$, fluoxetine $20 \mathrm{mg}$ daily & Not mentioned & No & No & N/A & None \\
\hline $\begin{array}{l}\text { Honca et al, } \\
2013(19)\end{array}$ & Case report & General inhalational & $\begin{array}{l}\text { Emergency caesarean } \\
\text { surgery }\end{array}$ & None & N/A & Not mentioned & BIS ${ }^{\circledast}$ monitor & Not mentioned & None \\
\hline $\begin{array}{l}\text { Pedrosa et al, } \\
2015(20)\end{array}$ & Case report & General intravenous & $\begin{array}{l}\text { Lumbar herniated disk } \\
\text { surgery }\end{array}$ & Modafinil $200 \mathrm{mg}$ daily & Yes & No & $\begin{array}{l}\text { BIS }{ }^{\circledast} \text { and entropy } \\
\text { monitors }\end{array}$ & 4 minutes & None \\
\hline
\end{tabular}

\section{Conclusions}

Nineteen case reports and one case series were included. We did not find clinical trials. The only review article found was written prior to the publishment of almost all case reports.

Excessive sleepiness, sleep paralysis and hypnagogic/hypnopompic hallucinations were reported in 3 narcoleptic patients after inhalational anesthesia. However, 6 reports of uneventful inhalational anesthesias were also found. Concerning intravenous anesthesia for narcoleptic patients, 6 case reports were found, showing no complications. A case series of 10 narcoleptic patients who underwent 27 general anesthesias showed that time for extubation did not differ from that observed in age and procedure match controls, during the same period.

Cataplexy episodes were reported in 2 narcoleptic patients undergoing spinal anesthesia and femoral nerve block. We found 2 case reports of epidural blocks and 1 case of an axillary brachial plexus block, with no complications.

Depth of anesthesia monitors were used in less than half of the patients. BIS ${ }^{\circledR}$ or entropy monitoring can be important to optimize anesthetic administration and to monitor a possible narcoleptic episode.

Benzodiazepines were administered preoperatively in four cases. Two of the patients had narcoleptic events intra ou post-operatively.

In the majority of cases, the patient's usual medication for narcolepsy was continued on the day of surgery, which may have contributed to a more rapid and complete recovery of consciousness.

Avoidance of sedative premedication, continuation of medication for narcolepsy on the day of surgery, use of short-acting anesthetic agents and depth of anesthesia monitoring may be beneficial measures in the management of narcoleptic patients.

\section{References}

1. Spector M, Bourke DL. Anesthesia, sleep paralysis, and 2. Pasteau F, Graftieaux JP, Gomis P, Scavarda D, Leon A. Problems
posed by posed by spinal anesthesia in a p
Anesth Reanim 1996;15:669-72 Anesth Reanim 1996,15:669-72
3. Mesa A, Dim AP, Frosth M. Na
Anesthesiology 2000:92:1194-6. 4. Hischer SP, Schmiesing CA, Guta CG, Brock-Utne JG. Genera stopped preoperatively? Anesth Analg. 2006 Jul; $103(1): 203-6$.

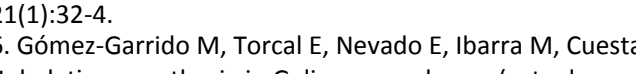

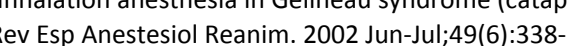
Vieito M, Hernández J, Hernández C, Arxer A. [Two additional cases of anesthesia in patients with narcolepsy]. Rev Esp Anestesiol Reanim 8. Pelíez R, Hortal $\mathrm{F}$, Bas cardiac surgery: can anesthesia with propofol and remife
safe? I Cardiothorac Vasc Anesth. 2004 Apr; 18(2):201-3. 9. Burrow B, Burkle C, Warner DO, Chini EN, Postoperative outcome
op patients with narcolepsy. A retrospective analysis. J Clin Anesth.

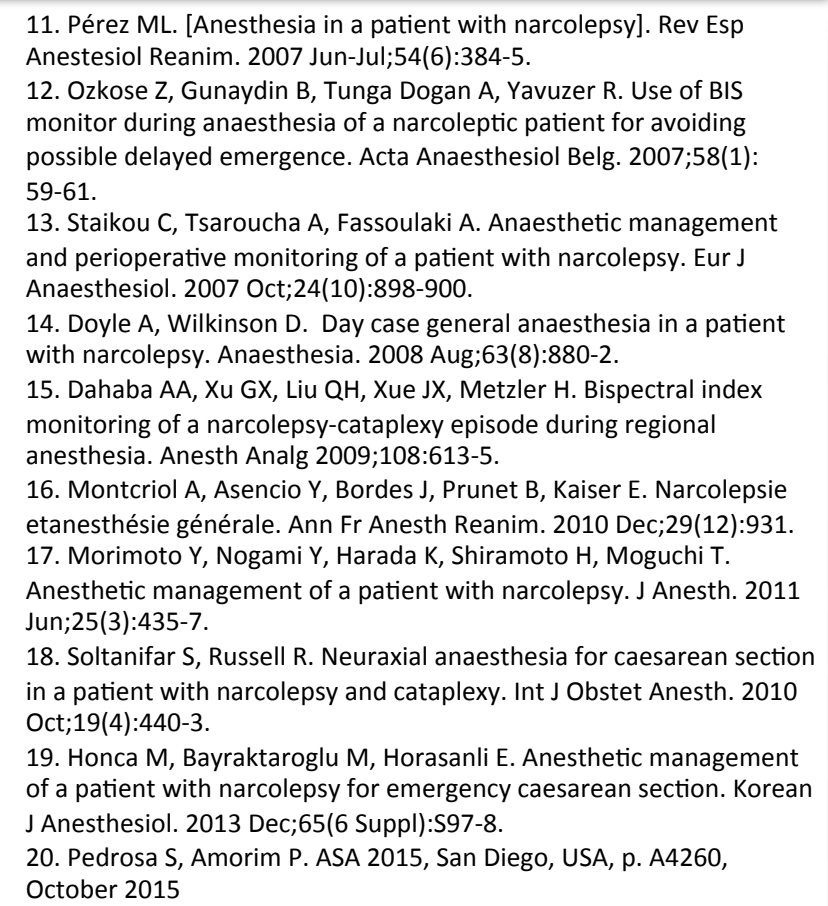

